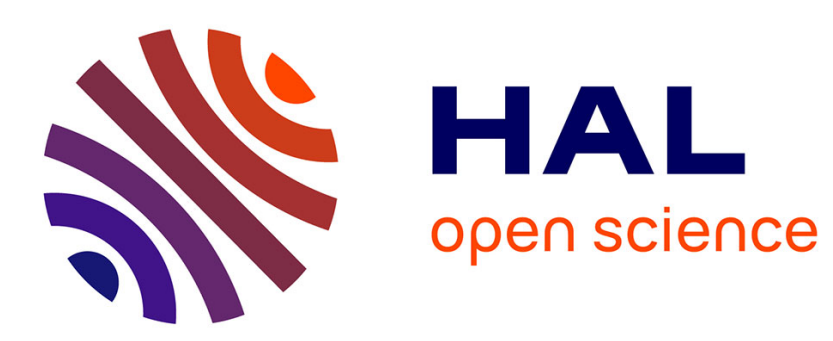

\title{
Maximal chains of closed prime ideals for discontinuous algebra norms on $\mathrm{C}(\mathrm{K})$
}

\author{
Jean Esterle
}

\section{To cite this version:}

Jean Esterle. Maximal chains of closed prime ideals for discontinuous algebra norms on $\mathrm{C}(\mathrm{K})$. Mathematical Proceedings of the Royal Irish Academy, 2012, 112A (2), pp.101-115. hal-00773663

\section{HAL Id: hal-00773663 \\ https://hal.science/hal-00773663}

Submitted on 14 Jan 2013

HAL is a multi-disciplinary open access archive for the deposit and dissemination of scientific research documents, whether they are published or not. The documents may come from teaching and research institutions in France or abroad, or from public or private research centers.
L'archive ouverte pluridisciplinaire HAL, est destinée au dépôt et à la diffusion de documents scientifiques de niveau recherche, publiés ou non, émanant des établissements d'enseignement et de recherche français ou étrangers, des laboratoires publics ou privés. 


\title{
Maximal chains of closed prime ideals for discontinuous algebra norms on $\mathcal{C}(K)$
}

\author{
J. Esterle
}

\begin{abstract}
Let $K$ be an infinite compact space, let $\mathcal{C}(K)$ be the algebra of continuous complex-valued functions of $K$, let $\mathcal{F}$ be a well-ordered chain of nonmaximal prime ideals of $\mathcal{C}(K)$, let $\mathcal{I}_{\mathcal{F}}$ be the smallest element of $\mathcal{F}$ and let $\mathcal{M}_{\mathcal{F}}$ be the unique maximal ideal of $\mathcal{C}(K)$ containing the elements of $\mathcal{F}$. Assuming the continuum hypothesis, we show that if $\left|\mathcal{C}(K) / \mathcal{I}_{\mathcal{F}}\right|=2^{\aleph_{0}}$, and if there exists a sequence $\left(\mathcal{G}_{n}\right)_{n \geq 1}$ of subsets of $\mathcal{F} \cup\left\{\mathcal{M}_{\mathcal{F}}\right\}$ stable under unions such that $\mathcal{F} \cup\left\{\mathcal{M}_{\mathcal{F}}\right\}=\cup_{n \geq 1} \mathcal{G}_{n}$, then there exists a discontinuous algebra norm $p$ on $\mathcal{C}(K)$ such that the set of all nonmaximal prime ideals of $\mathcal{C}(K)$ which are closed with respect to $p$ equals $\mathcal{F}$.
\end{abstract}

AMS classification: 46H40 (primary), 46J10, 03E50 (secondary)

\section{Introduction}

Let $K$ be an infinite compact space, and let $\mathcal{C}(K)$ denote the algebra of continuous complex valued functions on $K$. An algebra seminorm $\|\cdot\|$ on $\mathcal{C}(K)$ is a seminorm satisfying $\|f g\| \leq\|f\| g \|$ for every $f, g \in \mathcal{C}(K)$, and such a seminorm is said to be continuous if there exists $k>0$ such that $\|f\| \leq k\|f\|_{K}$ for every $f \in \mathcal{C}(K)$, where $\|f\|_{K}:=\max _{t \in K}|f(t)|$ denotes the usual norm on $\mathcal{C}(K)$. A classical result of Kaplansky [17] shows that if $\|$.$\| is any algebra norm on \mathcal{C}(K)$ we have

$$
\|f\| \geq\|f\|_{K} \text { for every } f \in \mathcal{C}(K)
$$

The existence of a discontinouous algebra seminorm on $\mathcal{C}(K)$, which is equivalent to the existence of a discontinuous algebra norm on $\mathcal{C}(K)$ and to the existence of a discontinuous homomorphism from $\mathcal{C}(K)$ into a Banach algebra, is the well-known Kaplansky's problem, which turns out to be undecidable in ZFC. Badé and Curtis obtained in [2] some partial continuity results, but H.G. Dales and the author [3], [10], [5] proved independently that if $2^{\aleph_{0}}=\aleph_{1}$, which means that the continuum hypothesis $(\mathrm{CH})$ is assumed, then discontinuous algebra seminorms exist on $\mathcal{C}(K)$ for every infinite compact space $K$. Those commutative Banach algebras $A$ for which a discontinuous homomorphism $\phi: \mathcal{C}(K) \rightarrow A$ 
does exist under $\mathrm{CH}$ were characterized in [11], see also [4]. On the other direction Solovay and Woodin constructed models of set theory including the axiom of choice and Martin's axiom in which all algebra seminorms on $\mathcal{C}(K)$ are continuous, see [6] for details. Notice that models of set theory in which $2^{\aleph_{0}}=\aleph_{2}$ and in which discontinuous algebra seminorms on $\mathcal{C}(K)$ exist for every infinite compact space $K$ were constructed independently by Frankiewicz- Zbierski and Woodin [14] [26].

The structure of closed ideals of $\mathcal{C}(K)$ for discontinuous algebra seminorms was investigated by A.M. Sinclair [24] and later, independently, by the author [8], who showed that the closure of an ideal is the intersection of all closed prime ideals which contain it. Also a chain of nonmaximal closed prime ideals is wellordered with respect to inclusion, see [8]. If $K$ is an $F$-space, which means that $f$ and $|f|$ generate the same ideal of $\mathcal{C}(K)$ for every $f \in \mathcal{C}(K)$, then the family $\operatorname{Prim}(q)$ of all nonmaximal prime ideals which are closed with respect to an algebra seminorm $q$ on $\mathcal{C}(K)$ is a finite union of well-ordered chains of nonmaximal prime ideals.

Pham [19], [20], [21] showed that the situation is much more complicated in the general case. We refer to the survey paper [12] for a discussion of his deep contributions and of problems which remain open.

This paper is part of a program intended to describe, assuming the continuum hypothesis, the families $\mathcal{U}$ of nonmaximal prime ideals of $\mathcal{C}(K)$ such that $\mathcal{U}=\operatorname{Prim}(q)$ for some algebra seminorm $q$ on $\mathcal{C}(K)$ (or, equivalently, for some algebra norm $q$ on $\mathcal{C}(K)$ ), where $\operatorname{Prim}(q)$ denotes the set of nonmaximal prime ideals of $\mathcal{C}(K)$ which are closed with respect to $q$. A neccessary condition is related to the notion of pseudo-finite family introduced by Pham in [19]: a family $\left(E_{\lambda}\right)_{\lambda \in \Lambda}$ of subsets of a set $E$ is said to be pseudofinite if the set $\left\{\mu \in \Lambda \mid x \notin E_{\mu}\right\}$ is finite for every $x \in \cup_{\lambda \in \Lambda} E_{\lambda}$. Using results of [8] and ideas from [19] and [21] it is possible to show that every sequence of elements of $\operatorname{Prim}(q)$ must contain a pseudofinite subsequence (this means that $\operatorname{Prim}(q)$ is "relatively compact" in the sense of [21]), which implies that every union of elements of $\operatorname{Prim}(q)$ is a finite union of prime ideals, see [21].

Also $\left\{I_{\zeta}\right\}_{\zeta<\omega}$ is a well-ordered family of elements of $\operatorname{Prim}(q)$, with $I_{\eta} \subsetneq I_{\zeta}$ for $\eta<\zeta$, then $\cup_{\zeta<\sigma} I_{\zeta} \in \operatorname{Prim}(q)$ if $[0, \sigma$ [ has no countable cofinal subset, since every sequence of elements of $\cup_{\eta<\zeta} I_{\eta}$ is contained in $\mathcal{I}_{\eta}$ for some $\eta<\zeta$.

Similarly if $\left(I_{\lambda}\right)_{\lambda \in \Lambda}$, is a pseudo-finite family of elements of $\operatorname{Prim}(q)$, with $I_{\lambda} \nsubseteq I_{\mu}$ for $\lambda \neq \mu$, and if $\Lambda$ is uncountable, then $\cup_{\lambda \in \Lambda} I_{\lambda} \in \operatorname{Prim}(q)$, since every sequence of elements of $\cup_{\lambda \in \Lambda} I_{\lambda}$ is contained in $I_{\lambda}$ for some $\lambda \in \Lambda$. We do not know whether these three necessary conditions are together sufficient for a family $\mathcal{U}$ of nonmaximal prime ideals of $\mathcal{C}(K)$ satisfying $\left|\mathcal{C}(K) / I_{\mathcal{U}}\right|=2^{\aleph_{0}}$ to be equal to $\operatorname{Prim}(q)$ for some algebra seminorm $q$ on $\mathcal{C}(K)$.

In this paper we will focus attention on chains of nonmaximal primes. Our main result is the fact that if a well-ordered chain $\left(I_{\zeta}\right)_{\zeta \leq \omega}$ of prime ideals of $\mathcal{C}(K)$, with $I_{\omega}$ maximal, is "almost stable under unions", and if $\left|\mathcal{C}(K) / I_{0}\right|=$ $2^{\aleph_{0}}$, then there exists a discontinuous algebra seminorm $q$ (or, equivalently, a discontinuous algebra norm $q$ ) on $\mathcal{C}(K)$ such that $\left\{I_{\zeta}: \zeta<\omega\right\}=\operatorname{Prim}(q)$. We do not know whether all maximal chains of closed prime are "almost stable 
under unions" in the sense of definition 1, but proposition 2.6 shows that the construction of a counterexample would require methods very different from the method used in the paper.

The author has known for many years that if $\left(I_{\zeta}\right)_{\zeta<\omega}$ is a chain of nonmaximal prime ideals of $\mathcal{C}(K)$, and if $\left|\mathcal{C}(K) / I_{0}\right|=2^{\aleph_{0}}$, then there exists an algebra seminorm $q$ on $\mathcal{C}(K)$ such that $\left\{I_{\zeta}: \zeta<\omega\right\} \subset \operatorname{Prim}(q)$, but was looking for a "natural" proof: it is known that given two prime ideals $I$ and $J$ of $\mathcal{C}(K)$ such that $I \subset J$ there exists a subalgebra $A_{J}$ of $\mathcal{C}(K) / I$ such that $\mathcal{C}(K) / I=A_{J} \oplus \pi(J)$, where $\pi: \mathcal{C}(K) \rightarrow \mathcal{C}(K) / I$ denotes the canonical surjection. It it were possible to construct a family $\left(A_{J}\right)_{J \supset I, J p r i m e}$ such that $A_{J_{2}} \subset A_{J_{1}}$ for $J_{1} \subset J_{2}$, then we hould have $P_{J_{1}} \circ P_{J_{2}}=P_{J_{2}} \circ P_{J_{1}}=P_{J_{2}}$, where $P_{J}$ denotes the projection of $\mathcal{C}(K) / I$ onto $A_{J}$ such that $\operatorname{Ker}\left(P_{J}\right)=\pi(J)$. Given any chain $\mathcal{F}$ of nonmaximal prime ideals of $\mathcal{C}(K)$ containing $I$ and any algebra norm $p$ on $\mathcal{C}(K) / I$ the formula

$$
q(f)=\max _{J \in \mathcal{F}}\left(p \circ P_{J} \circ \pi\right)(f)
$$

would define an algebra seminorm $q$ on $\mathcal{C}(K)$ such that $\mathcal{F} \subset \operatorname{Prim}(q)$. A variant of this formula using a suitable nonincreasing sequence of algebra norms on $\mathcal{C}(K) / I$ converging pointwise to zero would allow to prove directly our main result. Unfortunately, the author has been unable so far to construct such a family $\left(A_{J}\right)_{J \supset I, J p r i m e}$. So we present an indirect proof, based on the obvious existence of an analogous family for the "universal" algebra of power series $\mathbb{C}_{\omega_{1}}$ used in [9] and [10], see section 2.

We discuss at the end of the paper a natural extension of the main result which, if true, would allow to obtain a complete characterization (under $\mathrm{CH}$ ) of the continuity ideals associated to discontinuous homomorphisms from $\mathcal{C}(K)$.

\section{Algebra norms on the universal algebra $\mathbb{C}_{\omega_{1}}$ and chains of closed primes}

We introduce some objects used by the author in his construction of discontinuous homomorphisms from $\mathcal{C}(K)$. Let $\omega_{1}$ be the smallest uncountable ordinal. We denote by $S_{\omega_{1}} \subset\{0,1\}^{\omega_{1}}$ the set of all transfinite dyadic sequences $x=\left(x_{\zeta}\right)_{\zeta<\omega_{1}}$ for which there exists $\eta(x)<\omega_{1}$ such that $x_{\eta(x)}=1$ and such that $x_{\zeta}=0$ for every $\zeta>\eta(x)$.

Equiped with the lexicographic order, $S_{\omega_{1}}$ is a linearly ordered set, and a classical result of Sierpiński [23], see also [4] shows that every linearly ordered set of cardinal $\leq \aleph_{1}$ is order-isomorphic to a subset of $S_{\omega_{1}}$.

Denote by $G_{\omega_{1}} \subset S_{\omega_{1}}^{\mathbb{R}}$ the set of all real-valued functions $\phi$ on $S_{\omega_{1}}$ such that $\operatorname{Supp}(\phi):=\left\{s \in S_{\omega_{1}} \mid \phi(s) \neq 0\right\}$ is well-ordered and at most countable. For $\phi \in G_{\omega_{1}} \backslash\{0\}$, denote by $\rho(\phi)$ the smallest element of $\operatorname{Supp}(\phi)$. By definition, a nonzero element $\phi \in G_{\omega_{1}}$ is said to be strictly positive if $\phi(\rho(\phi))>0$. Equipped with the linear structure inherited from the linear structure of $S_{\omega_{1}}^{\mathbb{R}}, G_{\omega_{1}}$ is a 
linearly ordered real vector space, which contains a copy of every linearly ordered group of cardinal $\aleph_{1}$.

Now let $G$ be a linearly ordered group, and let $k$ be a field. We will denote by $\mathcal{F}(G, k)$ the set of all functions $f: G \rightarrow k$ such that $\operatorname{Supp}(f):=\{\tau \in G \mid f(\tau) \neq$ $0\}$ is well-ordered, and we set

$$
\mathcal{F}_{(1)}(G, k):=\left\{f \in \mathcal{F}(G, k)|| \operatorname{supp}(g) \mid \leq \aleph_{0}\right\} .
$$

Now let $f, g \in \mathcal{F}(G, k)$, and let $\tau \in G$. If $\tau \notin \operatorname{Supp}(f)+\operatorname{Supp}(g):=\{\alpha+$ $\beta\}_{\alpha \in \operatorname{Supp}(f), \beta \in \operatorname{Supp}(g)}$, set $(f g)(\tau)=0$. Otherwise set

$$
(f g)(\tau)=\sum_{\substack{\alpha \in S u p p(f), \beta \in \operatorname{Supp}(g) \\ \alpha+\beta=\tau}} f(\alpha) g(\beta) .
$$

Then $f g$ is well-defined, since the set $\{(\alpha, \beta) \in \operatorname{Supp}(f) \times \operatorname{Supp}(g) \mid \alpha+\beta=$ $\tau\}$ is finite for every $\tau \in \operatorname{Supp}(f)+\operatorname{Supp}(g)$, and $f g \in \mathcal{F}(G, k)$. In fact Hahn observed in 1907 in [16] that $\mathcal{F}(G, k)$ is a field. Set $v(f)=\inf (\operatorname{Supp}(f))$ for $f \in \mathcal{F}(G, k) \backslash\{0\}$. Then $v$ is a valuation on the field $\mathcal{F}(G, k)$, and the valued field $\mathcal{F}(G, k)$ is maximal: if $U$ is a field containing $\mathcal{F}(G, k)$, and if $w$ is a valuation on $U$ with values in $G$ such that $w(f)=v(f)$ for every $f \in \mathcal{F}(G, k) \backslash\{0\}$, then $U=\mathcal{F}(G, k)$.

Mac Lane showed in [18] that $\mathcal{F}(G, k)$ is algebraically closed if $k$ is algebraically closed and if the equation $n t=\tau$ has a solution in $G$ for every $\tau \in G$ and every integer $n \geq 2$. In particular, the fields $\mathcal{F}\left(G_{\omega_{1}}, \mathbb{C}\right)$ and $\mathcal{F}_{(1)}\left(G_{\omega_{1}}, \mathbb{C}\right)$ are algebraically closed.

We set, with the convention $v(0)=+\infty>\tau$ for every $\tau \in \mathbf{G}$,

$$
\mathbb{C}_{\omega_{1}}:=\left\{f \in \mathcal{F}_{(1)}\left(G_{\omega_{1}}, \mathbb{C}\right) \mid v(f) \geq 0\right\}, \mathcal{M}_{\omega_{1}}:=\left\{f \in \mathcal{F}_{(1)}\left(G_{\omega_{1}}, \mathbb{C}\right) \mid v(f)>0\right\},
$$

so that the radical complex algebra $\mathcal{M}_{\omega_{1}}$ is the unique maximal ideal of the commutative unital complex algebra $\mathbb{C}_{\omega_{1}}$. The algebra $\mathbb{C}_{\omega_{1}}$ is universal: if the continuum hypothesis is assumed then $\mathbb{C}_{\omega_{1}}$ contains a copy of every commutative unital complex algebra of cardinality $2^{\aleph_{0}}$ which is an integral domain and possesses a character.

Let $r \in \mathcal{M}_{\omega_{1}}$. Since the field of fractions of $\mathbb{C}_{\omega_{1}}$ is algebraically closed, the equation $y^{n}=1+r$ has $n$ solutions of the form $e^{\frac{2 i k}{n} \pi}\left(1+r_{n}\right) \in \mathbb{C}_{\omega_{1}}$ where $0 \leq k \leq n-1$. Set

$$
(1+r)^{\frac{1}{n}}=1+r_{n}
$$

Now let $p$ be any algebra seminorm on $\mathbb{C}_{\omega_{1}}$. Let $R$ be the completion of $\mathcal{M}_{\omega_{1}} / \operatorname{Kerp}$ with respect to $p$, and let $\pi=\mathbb{C}_{\omega_{1}} \rightarrow \mathbb{C}_{\omega_{1}} / \operatorname{Ker}(p)$ be the canonical surjection. Then $A:=R \oplus \mathbb{C} .1$ is the completion of $\mathbb{C}_{\omega_{1}}$ with respect to $p$. Since $R$ is a commutative radical algebra, the series $\sum_{n=1}^{\infty}(-1)^{n+1} \frac{\pi(r)^{n}}{n}$ converges in $R$. Set $a:=\sum_{n=1}^{\infty}(-1)^{n+1} \frac{\pi(r)^{n}}{n}$, so that $1+\pi(r)=\exp (a)$. We have 
$0=\exp (a)-\left(1+\pi\left(r_{n}\right)\right)^{n}=\left(\exp (a / n)-1-\pi\left(r_{n}\right)\right) \sum_{j=0}^{n-1} \exp (j a / n)\left(1+\pi\left(r_{n}\right)\right)^{n-j-1}$

We have $\chi\left(\sum_{j=0}^{n-1} \exp (j a / n)\left(1+\pi\left(r_{n}\right)\right)^{n-j-1}\right)=n$, where $\chi$ denotes the unique character on $A$, and so $\sum_{j=0}^{n-1} \exp (j a / n)\left(1+\pi\left(r_{n}\right)\right)^{n-j-1}$ is invertible in $A$. We obtain

$$
1+\pi\left(r_{n}\right)=\exp (a / n), \quad \lim _{n \rightarrow \infty} p\left(r_{n}\right)=0 .
$$

The author's construction of a discontinuous homomorphism of $\mathcal{C}(K)$ was based on the existence of algebra norms on $\mathbb{C}_{\omega_{1}}$, suggested by Allan's embedding of $\mathbb{C}[[X]]$, the algebra of all formal power series in one variable, into some Banach algebras [1](Dales and Woodin discuss in [7] the normability of algebras of formal power series larger than $\mathbb{C}_{\omega_{1}}$, which remains an open problem). We will need a precise form of this result, given by the following theorem.

Theorem 2.1 There exists a sequence $\left(\|\cdot\|_{n}\right)_{n \geq 1}$ of algebra norms on $\mathbb{C}_{\omega_{1}}$ satisfying the following properties

(i) $\|u\|_{n+1} \leq\|u\|_{n}$ for every $u \in \mathbb{C}_{\omega_{1}}$, and $\lim _{n \rightarrow \infty}\|u\|_{n}=0$ for every $u \in \mathcal{M}_{\omega_{1}}$,

(ii) for every $\tau \in G_{\omega_{1}}^{+}$and every $r \in \mathcal{M}_{\omega_{1}}$, we have

$$
\begin{gathered}
\lim _{n \rightarrow \infty}\left\|X^{\tau / n}\right\|_{1}=1 \text {, and } \\
\lim _{n \rightarrow \infty}\left\|w-w X^{\tau / n}(1+r)^{\frac{1}{n}}\right\|_{1}=1 \text { for every } w \in \mathcal{M}_{\omega_{1}} .
\end{gathered}
$$

Proof: Denote by $\mathcal{A}(\mathbb{D})$ the usual disc algebra of functions holomorphic on the open unit disc $\mathbb{D}$ which have a continuous extension to the closed unit disc $\overline{\mathbb{D}}$, which is a Banach algebra with respect to the norm $\|f\|:=\max _{|z| \leq 1}|f(z)|=$ $\max _{|z|=1}|f(z)|$. Set $M=\{f \in \mathcal{A}(\mathbb{D}) \mid f(1)=0\}$, and set $\Omega:=\left\{f \in M \mid[f M]^{-}=\right.$ $M\}$. Since $M$ possesses a bounded approximate identity, it follows from [10] that there exists a one-to-one map $\delta: G_{\omega_{1}}^{+} \rightarrow \Omega \cup\{1\}$ such that $\delta(\sigma+\tau)=\delta(\sigma) \delta(\tau)$ for $\sigma \in G_{\omega_{1}}^{+}, \tau \in G_{\omega_{1}}^{+}$, and such that $\delta(\sigma) \in \Omega$ for $\sigma>0$, with $\delta(\tau)(0)>0$ for every $\tau \in G_{\omega_{1}}^{+}$.

Set $\theta_{m}(z)=\exp \left(\frac{z+1}{m(z-1)}\right)$ for $|z| \leq 1, z \neq 1$, so that $\theta_{m}$ is an inner function which is continuous on $\overline{\mathbb{D}} \backslash\{1\}$. Then $\theta_{m} M$ is a closed ideal of $\mathcal{A}(\mathbb{D})$ contained in $M$, the quotient algebra $M / \theta_{m} M$ is radical, and $\cup_{m \geq 1} \theta_{m} M$ is dense in $M$. Denote by $\pi_{m}: \mathcal{A}(\mathbb{D}) / \theta_{1} M \rightarrow \mathcal{A}(\mathbb{D}) / \theta_{m} M$ the canonical surjection. It follows from [9] that there exists a one-to-one homomorphism $\phi: \mathbb{C}_{\omega_{1}} \rightarrow \mathcal{A}(\mathbb{D}) / \theta_{1} M$ such that $\phi\left(X^{\tau}\right)=\left(\pi_{1} \circ \delta\right)\left(X^{\tau}\right)$ for $\tau \in G_{\omega_{1}}^{+}$. Set, for $u \in \mathbb{C}_{\omega_{1}}, n \geq 1$,

$$
\|u\|_{n}=\left\|\left(\pi_{n} \circ \phi\right)(u)\right\| .
$$


Set $\pi=\pi_{1}$, let $u \in \mathbb{C}_{\omega_{1}} \backslash\{0\}$, and set $\tau=v(u)$. We have $u=X^{\tau}(\lambda+w)$, where $\lambda \in \mathbb{C} \backslash\{0\}, w \in \mathcal{M}_{\omega_{1}}$, and so $\phi(u)=\pi\left(\delta\left(X^{\tau}\right)\right)(\lambda+\phi(w))$. Clearly, $\phi(w) \in \pi(M)$, and so $\pi_{n}(\lambda 1+\phi(w))$ is invertible in $\mathcal{A}(\mathbb{D}) / \theta_{n} M,\left(\pi_{n} \circ \phi\right)(u)$ generates a dense ideal of $M / \theta_{n} M$ and $\left(\pi_{n} \circ \phi\right)(u) \neq 0$. So $\|\cdot\|_{n}$ is an algebra norm on $\mathbb{C}_{\omega_{1}}$. Since $\theta_{n} M \subset \theta_{n+1} M$ for $n \geq 1$, and since $\cup_{n \geq 1} \theta_{n} M$ is dense in $M$, the sequence $\left(\|\cdot\|_{n}\right)_{n \geq 1}$ satisfies (i).

Since $\delta(\tau)$ is an outer function, there exists a function $f$ analytic on $\mathbb{D}$ and continuous on $\overline{\mathbb{D}} \backslash\{1\}$ such that $\delta(\tau)(z)=\exp (f(z))$ for $|z| \leq 1, z \neq 1$, and we can choose $f$ so that $f(0) \in \mathbb{R}$. Set $g_{n}(z)=\exp \left(\frac{f(z)}{n}\right)$ for $m \geq 1$, with the convention $g_{n}(1)=0$. Then $g_{n} \in \mathcal{A}(\mathbb{D})$, and $g_{n}^{n}=\delta(\tau)$. Since $g_{n}(0)>0$ and $\delta\left(\frac{\tau}{n}\right)(0)>0$, we have $g_{n}=\delta\left(\frac{\tau}{n}\right)=\phi\left(X^{\frac{\tau}{n}}\right)$.

Since $f(z) / n \rightarrow 0$ uniformly on every compact subset of $\overline{\mathbb{D}} \backslash\{1\}$ as $n \rightarrow \infty$, we have $\lim _{n \rightarrow \infty}\left\|h-h g_{n}\right\|=0$ for every $h \in M$. Hence $\lim _{n \rightarrow \infty}\left\|w-w X^{\frac{\tau}{n}}\right\|_{1}=0$ for every $w \in \mathcal{M}_{\omega_{1}}$. The fact that $\lim _{n \rightarrow \infty}\left\|w-w X^{\frac{\tau}{n}}(1+r)^{\frac{1}{n}}\right\|_{1}=1$ for $r \in \mathcal{M}_{\omega_{1}}$ follows then from (2).

We will use the following easy lemma.

Lemma 2.2 Let $\mathcal{P}_{\omega_{1}}$ be the set of all prime ideals of $\mathbb{C}_{\omega_{1}}$. For $I \in \mathcal{P}_{\omega_{1}}$, set $W_{I}:=\{v(u): u \in I \backslash\{0\}\}$, and for $u \in \mathbb{C}_{\omega_{1}}$ define $\theta_{I}(u): G_{\omega_{1}}^{+} \rightarrow \mathbb{C}$ by the formulae

$$
\begin{cases}\theta_{I}(u)(\tau)=0 & \text { if } \tau \in W_{I} \\ \theta_{I}(u)(\tau)=u(\tau) & \text { if } \tau \notin W_{I}\end{cases}
$$

Then $\theta_{I}(u) \in \mathbb{C}_{\omega_{1}}, \theta_{I}: \mathbb{C}_{\omega_{1}} \rightarrow \mathbb{C}_{\omega_{1}}$ is an algebra homomorphism, and we have the following properties:

(i) For every $I \in \mathcal{P}_{\omega_{1}}, \operatorname{Ker}\left(\theta_{I}\right)=I, u-\theta_{I}(u) \in I$ for $u \in \mathbb{C}_{\omega_{1}}, \theta_{I}\left(X^{\tau}\right)=X^{\tau}$ for $\tau \in G_{\omega_{1}}^{+} \backslash W_{I}$, and $\left[\theta_{I}(1+r)\right]^{1 / n}=\theta_{I}\left((1+r)^{1 / n}\right)$ for $r \in \mathcal{M}_{\omega_{1}}, n \geq 1$.

(ii) If $I_{1}, I_{2} \in \mathcal{P}_{\omega_{1}}$, and if $I_{1} \subseteq I_{2}$, then $\theta_{I_{2}} \circ \theta_{I_{1}}=\theta_{I_{1}} \circ \theta_{I_{2}}=\theta_{I_{2}}$.

(iii) If $u \in \mathbb{C}_{\omega_{1}}, I_{1} \in \mathcal{P}_{\omega_{1}}, I_{2} \in \mathcal{P}_{\omega_{1}}$, and if $\theta_{I_{1}}\left(u^{m}\right)=\theta_{I_{2}}\left(u^{m}\right)$ for some $m \geq 1$, then $\theta_{I_{1}}(u)=\theta_{I_{2}}(u)$.

(iv) If $\mathcal{F} \subset \mathcal{P}_{\omega_{1}}$ is well-ordered with respect to inclusion, then the set $\left\{\theta_{I}(u)\right\}_{I \in \mathcal{F}}$ is finite for every $u \in \mathbb{C}_{\omega_{1}}$.

Proof: Since $\operatorname{Supp}\left(\theta_{I}(u)\right) \subset \operatorname{Supp}(u)$, we have $\theta_{I}(u) \in \mathbb{C}_{\omega_{1}}$ for every $u \in \mathbb{C}_{\omega_{1}}$. Clearly, $\theta_{I}\left(X^{\tau}\right)=X^{\tau}$ for every $\tau \in G_{\omega_{1}}^{+} \backslash W_{I}$. Also if $r \in \mathcal{M}_{\omega_{1}}, n \geq 1$, we have $\theta_{I}(1+r)-1=\theta_{I}(r) \in \mathcal{M}_{\omega_{1}}$, and $\theta_{I}\left([1+r]^{1 / n}\right)^{n}=\theta_{I}(1+r)$, which shows that $\left[\theta_{I}(1+r)\right]^{1 / n}=\theta_{I}\left([1+r]^{1 / n}\right)$.

The map $\theta_{I}: \mathbb{C}_{\omega_{1}} \rightarrow \mathbb{C}_{\omega_{1}}$ is linear. Each $u \in \mathbb{C}_{\omega_{1}} \backslash\{0\}$ can be written under the form $u=X^{v(u)} w$, where $w \in \operatorname{Inv}\left(\mathbb{C}_{\omega_{1}}\right)$, and so $u \in I$ if and only if $v(u) \in W_{I}$, and $G_{\omega_{1}} \backslash W_{I}$ is stable under sums. This shows that $\theta_{I}$ is an algebra homomorphism, that $\operatorname{Ker}\left(\theta_{I}\right)=I$ and that $u-\theta_{I}(u) \in I$ every $u \in \mathbb{C}_{\omega_{1}}$. It follows then from the definition of $\theta_{I_{1}}$ and $\theta_{I_{2}}$ that $\theta_{I_{2}} \circ \theta_{I_{1}}=\theta_{I_{1}} \circ \theta_{I_{2}}=\theta_{I_{2}}$ if $I_{1} \subseteq I_{2}$. 
Now assume that $\theta_{I_{1}}\left(u^{m}\right)=\theta_{I_{2}}\left(u^{m}\right)$ for some $m \geq 1$, with $I_{2} \subset I_{1}$. If $u \in I_{1}$, there is nothing to prove. If $u \notin I_{1}$, then $u^{m-1} \notin I_{1}$, and $\sum_{j=0}^{m-1} \theta_{I_{1}}\left(u^{j}\right) \theta_{I_{2}}\left(u^{m-j}\right)-$ $m u^{m-1} \in I_{1}$, and so $\sum_{j=0}^{m-1} \theta_{I_{1}}\left(u^{j}\right) \theta_{I_{2}}\left(u^{m-j}\right) \neq 0$. We have

$$
\left.0=\theta_{I_{1}}\left(u^{m}\right)-\theta_{I_{2}}\left(u^{m}\right)=\left(\theta_{I_{1}}\right)(u)-\theta_{I_{2}}(u)\right) \sum_{j=0}^{m-1} \theta_{I_{1}}\left(u^{j}\right) \theta_{I_{2}}\left(u^{m-j}\right),
$$

and so $\theta_{I_{1}}(u)=\theta_{I_{2}}(u)$.

Let $\mathcal{F}$ be a well-ordered chain of elements of $\mathcal{P}_{\omega_{1}}$, let $\left(I_{n}\right)_{n \geq 1}$ be a strictly increasing family of elements of $\mathcal{F}$, set $I:=\cup_{n \geq 1} I_{n}$, and let $u \in \mathcal{C}_{\omega_{1}}$. Since $u-\theta_{I}(u) \in I$, there exists $m \geq 1$ such that $u-\bar{\theta}_{I}(u) \in I_{m}$. Hence for $n \geq m$ we have

$$
0=\theta_{I_{n}}\left(u-\theta_{I}(u)\right)=\theta_{I_{n}}(u)-\left(\theta_{I_{n}} \circ \theta_{I}\right)(u)=\theta_{I_{n}}(u)-\theta_{I}(u) .
$$

Since $\mathcal{F}$ is well-ordered, this shows that the set $\left\{\theta_{I}(u)\right\}_{I \in \mathcal{F}}$ is finite for every $u \in \mathbb{C}_{\omega_{1}}$.

We will need the following simple observation.

Lemma 2.3 Let $A$ be a unital subalgebra of $\mathbb{C}_{\omega_{1}}$ which possesses the following properties

(i) If $a, b \in A$, and if $a^{2} \notin b A$, then $b^{2} \in a A$,

(ii) the equation $x^{n}=a$ has a solution in $A$ for every $a \in A$ and every $n \geq 1$.

If $I$ is a prime ideal of $A$, denote by $I^{-}$the the ideal of $\mathbb{C}_{\omega_{1}}$ generated by $I$, and denote by $I^{+}$the union of all ideals $J$ of $\mathbb{C}_{\omega_{1}}$ such that $J \cap(A \backslash I)=\emptyset$. Then $I^{-} \subset I^{+}, I^{-}$and $I^{+}$are prime ideals of $\mathbb{C}_{\omega_{1}}$, and $I=I^{-} \cap A=I^{+} \cap A$.

Proof: Since the ideals of $\mathbb{C}_{\omega_{1}}$ form a chain, $I^{+}$is an ideal of $\mathbb{C}_{\omega_{1}}$, which is maximal among all ideals $J$ of $\mathbb{C}_{\omega_{1}}$ such that $J \cap(A \backslash I)=\emptyset$. Since $A \backslash I$ is stable under products, a standard result of elementary algebra shows that $I^{+}$is prime.

Let $u \in I^{-} \backslash\{0\}$. We have $u=a_{1} b_{1}+\ldots+a_{m} b_{m}$, where $a_{1}, \ldots, a_{m} \in I$ and where $b_{1}, \ldots, b_{m} \in \mathbb{C}_{\omega_{1}}$.

Let $j_{0} \in\{1, \ldots, m\}$ be such that $v\left(a_{j_{0}} b_{j_{0}}\right)=\min _{1 \leq j \leq m} v\left(a_{j} b_{j}\right)$. Then $v(u) \geq$ $v\left(a_{j_{0}} b_{j_{0}}\right)=v\left(a_{j_{0}}\right)+v\left(b_{j_{0}}\right) \geq v\left(a_{j_{0}}\right)$, and so $v\left(u a_{j_{0}}^{-1}\right) \geq 0, u a_{j_{0}}^{-1} \in \mathbb{C}_{\omega_{1}}$, and $u \in a_{j_{0}} \mathbb{C}_{\omega_{1}}$. Hence $I^{-}=\cup_{a \in I} a \mathbb{C}_{\omega_{1}}$.

Let $u \in \mathbb{C}_{\omega_{1}}$ satisfying $u^{n} \in I^{-}$for some $n \geq 1$, and let $a \in I$ and $b \in \mathbb{C}_{\omega_{1}}$ such that $u^{n}=a b$. There exists $c \in A$ and $d \in \mathbb{C}_{\omega_{1}}$ such that $c^{n}=a$ and $d^{n}=b$, and $u^{n}=c^{n} d^{n}$. Hence $u=\lambda c d$ for some $\lambda \in \mathbb{C}$ and $u \in c \mathbb{C}_{\omega_{1}} \subset I^{-}$, which shows that $I^{-}$is semiprime. Hence $I^{-}$is prime since the ideals of $\mathbb{C}_{\omega_{1}}$ form a chain.

Let $a \in A \backslash I$, and let $b \in I$. There exists $c \in A$ such that $c^{4}=b$, and $c \in I$. Then $a^{2} \notin c A$ since $a^{2} \notin I$, and so $c^{2} \in a A$ and $v(b)=2 v\left(c^{2}\right)>v\left(c^{2}\right) \geq v(a)$, 
which shows that $a \notin b \mathbb{C}_{\omega_{1}}$. Hence $a \notin I^{-}$. This shows that $I^{-} \cap A=I$, and $I^{-} \subset I^{+}$. Since $I^{+} \cap A \subset I$, we have $I^{-} \cap A=I^{+} \cap A=I$.

Notice that It follows from (i) that the prime ideals of $A$ form a chain, which is also a consequence of the fact that $I=I^{-} \cap A$ for every prime ideal $I$ of $A$. Hence $\cup_{\lambda \in \Lambda} I_{\lambda}$ is a prime ideal of $A$ for every family $\left(I_{\lambda}\right)_{\lambda \in \Lambda}$ of prime ideals of $A$. Since $I^{-}=\cup_{a \in I} a \mathbb{C}_{\omega_{1}}$, we have $\left[\cup_{\lambda \in \Lambda} I_{\lambda}\right]^{-}=\cup_{\lambda \in \Lambda} I_{\lambda}^{-}$.

It follows from the definition of the ideals $I^{+}$that $\cup_{\lambda \in \Lambda} I_{\lambda}^{+} \subset\left[\cup_{\lambda \in \Lambda} I_{\lambda}\right]^{+}$. But if there exists a sequence $\left(I_{n}\right)_{n \geq 1}$ of nonmaximal prime ideals of A such that $\mathcal{M}=\cup_{n \geq 1} I_{n}$, we have $\cup_{n \geq 1} I_{n}^{+} \subsetneq \mathcal{M}_{\omega_{1}}=\left[\cup_{n \geq 1} I_{n}\right]^{+}$, since the set of strictly positive elements of $G_{\omega_{1}}$ has no countable coinitial subset.

If $A$ is a family of linear subspaces on a complex linear space $E$ we will denote by $\mathcal{U}(A)$ the set of all unions of subfamilies of $A$, and we will denote by $\mathcal{V}(A)$ the set of all linear subspaces of $E$ which belong to $\mathcal{U}(A)$. We will say that $A$ is stable under unions if $\mathcal{V}(A)=A$. We will need the following notion.

Definition 1 Let $E$ be a linear space and let $\mathcal{F}$ be a family of linear subspaces of $E$. We will say that the family $\mathcal{F}$ is almost stable under unions if there exists a sequence $\left(\mathcal{F}_{n}\right)_{n \geq 1}$ of subfamilies of $\mathcal{F}$ such that $\cup_{n \geq 1} \mathcal{V}\left(\mathcal{F}_{n}\right)=\mathcal{F}$.

A countable family of linear subspaces of $E$ is indeed almost stable under unions. Also, since $\mathcal{V}(\mathcal{V}(A))=\mathcal{V}(A)$ for every family $A$ of linear subspaces of $E$, we see that if $\mathcal{F}$ is almost stable under unions there exists a sequence $\left(\mathcal{F}_{n}\right)_{n \geq 1}$ of subfamilies of $\mathcal{F}$ stable under unions such that $\mathcal{F}=\cup_{n \geq 1} \mathcal{F}_{n}$.

Notice that if $\mathcal{F}$ is almost stable under unions, and if $\mathcal{G}$ is a chain of elements of $\mathcal{F}$ which doest not admit any countable cofinal subset, then $\mathcal{G} \cap \mathcal{F}_{n}$ is cofinal in $\mathcal{G}$ for some $n \geq 1$, and so $\cup\{L: L \in \mathcal{G}\} \in \mathcal{F}$.

The main results of the paper are a direct consequence of the following lemma.

Lemma 2.4 Let $A$ be a unital subalgebra of $\mathbb{C}_{\omega_{1}}$ satisfying conditions (i) and (ii) of lemma 2.3, let $\mathcal{M}$ be the unique maximal ideal of $A$, let $\mathcal{F}$ be a well-ordered family of elements of nonmaximal prime ideals of $A$, and set $\tilde{\mathcal{F}}:=\left\{I^{+}\right\}_{I \in \mathcal{F} \text {. }}$ Then there exists an algebra norm $q$ on $\mathbb{C}_{\omega_{1}}$ such that every element of $\tilde{\mathcal{F}}$ is closed in $\mathbb{C}_{\omega_{1}}$ with respect to $q$, so that every element of $\mathcal{F}$ is closed in $A$ with respect to $q$.

If, further, $\mathcal{F} \cup\{\mathcal{M}\}$ is almost stable under unions, and if the ideals of $A$ closed with respect to any algebra seminorm on $A$ are prime, then there exists an algebra seminorm on $\mathbb{C}_{\omega_{1}}$ such that the set of all nonmaximal prime ideals of $A$ which are closed with respect to $q$ equals $\mathcal{F}$.

Proof: Let $p$ be any algebra norm on $\mathbb{C}_{\omega_{1}}$, and set $q(u)=\max _{J \in \tilde{\mathcal{F}}} p\left(\theta_{J}(u)\right)$ for $u \in \mathbb{C}_{\omega_{1}}$, where $\left(\theta_{J}\right)_{J \in \mathcal{P}_{\omega_{1}}}$ is the family of homomorphisms constructed in lemma 2.2. Since $\tilde{\mathcal{F}}$ is well-ordered with respect to inclusions, it follows from lemma 2.2 (iv) that $q$ is well-defined, and, obviously, every element of $\tilde{\mathcal{F}}$ is closed in with respect to $q$, so that every $I \in \mathcal{F}$ is closed in $A$ with respect to $q$, since it follows from lemma 2.3 that $I=I^{+} \cap A$. 
Now assume that $\mathcal{F} \cup\{\mathcal{M}\}$ is almost stable under unions, and let $\left(\|\cdot\|_{n}\right)_{n \geq 1}$ be a sequence of algebra norms on $\mathbb{C}_{\omega_{1}}$ satisfying the conditions of theorem 2.1.

We can write $\mathcal{F} \cup\{\mathcal{M}\}=\left\{I_{\zeta}\right\}_{\zeta \leq \omega}$, and there exists a sequence $\left(\Omega_{n}\right)_{n \geq 1}$ of subsets $[0, \omega]$ satisfying $[0, \omega]=\cup_{n \geq 1} \Omega_{n}$ such that $\mathcal{F}_{n}:=\left\{I_{\zeta}\right\}_{\zeta \in \Omega_{n}}$ is stable under unions for every $n \geq 1$. For $\zeta \leq \omega$, let $n(\zeta)$ be the smallest positive integer such that $\zeta \in \Omega_{n}$, and set, for $u \in \mathbb{C}_{\omega_{1}}$,

$$
q_{\zeta}(u)=\left\|\theta_{I_{\zeta}^{+}}(u)\right\|_{n(\zeta)},
$$

so that $q_{\zeta}$ is an algebra seminorm on $\mathbb{C}_{\omega_{1}}$ such that $\operatorname{Ker}\left(q_{\zeta}\right)=I_{\zeta}^{+}$. It follows again from lemma 2.2 that the set $\left\{\theta_{I_{\zeta}^{+}}(u)\right\}_{\zeta<\omega}$ is finite for every $u \in \mathbb{C}_{\omega_{1}}$. Hence the set $\left\{q_{\zeta}(u)\right\}_{\zeta<\omega}$ has a largest element for $u \in \mathbb{C}_{\omega_{1}}$. Now set

$$
q_{\mathcal{F}}(u)=\max _{\zeta<\omega} q_{\zeta}(u) .
$$

We see again that every element of $\tilde{\mathcal{F}}$ is closed in $\mathbb{C}_{\omega_{1}}$ with respect to $q_{\mathcal{F}}$, so that every element of $\mathcal{F}$ is closed in $A$ with respect to $q_{\mathcal{F}}$.

Let $u \in \mathcal{M}$, and let $I_{\sigma}$ be the smallest element of $\mathcal{F} \cup\{\mathcal{M}\}$ containing $u$. We want to show that $u \mathbb{C}_{\omega_{1}}$ is dense in $I_{\sigma}^{+}$and that $u A$ is dense in $I_{\sigma}$ with respect to $q_{\mathcal{F}}$. We can restrict attention to the case where $u=X^{\tau}(1+r)$ for some strictly positive $\tau \in G_{\omega_{1}}$ and some $r \in \mathcal{M}_{\omega_{1}}$. Set $u_{n}=X^{\frac{\tau}{n}}(1+r)^{\frac{1}{n}}$ for $n \geq 1$. Since the complex algebra $A$ satisfies condition (ii) of lemma 2.3 , we have $u_{n} \in A$, and since the ideals of $A$ which are closed with respect to $q_{\mathcal{F}}$ are prime, $u_{n}$ belongs to the closure of $u A$ in $A$ with respect to $q_{\mathcal{F}}$ for every $n \geq 1$.

Let $a \in I_{\sigma}^{+}$. Then there exists a finite family $\left\{\zeta_{1}, \ldots, \zeta_{k}\right\}$ of elements of $[0, \sigma[$ such that for every $\zeta \in\left[0, \sigma\right.$ there exists $j \leq k$ satisfying $\theta_{I_{\zeta}}(a)=\theta_{I_{\zeta_{j}}}(a)$. It follows from lemma 2.2 (i) that we have

$$
\theta_{I_{\zeta}}\left(X^{\frac{\tau}{n}} a\right)=X^{\frac{\tau}{n}} \theta_{I_{\zeta}}(a)=X^{\frac{\tau}{n}} \theta_{I_{\zeta_{j}}}(a)=\theta_{I_{\zeta_{j}}}\left(X^{\frac{\tau}{n}} a\right) .
$$

Hence $q_{\mathcal{F}}\left(a-X^{\frac{\tau}{n}} a\right)=\max _{\zeta<\sigma} q_{\zeta}\left(a-X^{\frac{\tau}{n}} a\right)=\max _{j \leq k} q_{\zeta_{j}}\left(a-X^{\frac{\tau}{n}} a\right)$. We obtain

$$
\lim \sup _{n \rightarrow+\infty} q_{\mathcal{F}}\left(a-X^{\frac{\tau}{n}} a\right) \leq \lim _{n \rightarrow+\infty} \max _{j \leq k}\left\|\theta_{I_{\zeta_{j}}}(a)-X^{\frac{\tau}{n}} \theta_{I_{\zeta_{j}}}(a)\right\|_{1}=0
$$

It follows from $(2)$ that $\lim _{n \rightarrow+\infty} q_{\mathcal{F}}\left((1+r)^{\frac{1}{n}}-1\right)=0$, and we have

$$
\lim _{n \rightarrow+\infty} q_{\mathcal{F}}\left(a-a u_{n}\right)=0,
$$

which shows that $u \mathbb{C}_{\omega_{1}}$ is dense in $I_{\sigma}^{+}$and $u A$ is dense in $I_{\sigma}$ with respect to $q_{\mathcal{F}}$.

Now let $I$ be a prime ideal of $A$ which is closed with respect to $q_{\mathcal{F}}$, and let $\sigma$ be the smallest element of $[0, \omega]$ such that $I_{\sigma}$ contains $I$. Since the prime ideals of $A$ form a chain, we have $I_{\zeta} \subsetneq I$ for every $\zeta<\sigma$.

If $\cup_{\zeta<\sigma} I_{\zeta} \subsetneq I$, let $u \in I \backslash \cup_{\zeta<\sigma} I_{\zeta}$. Then $\sigma$ is the smallest element of $[0, \omega]$ such that $I_{\sigma}$ contains $u$, and so $u A$ is dense in $I_{\sigma}$ with respect to $q_{\mathcal{F}}$, which shows that $I=I_{\sigma} \in \mathcal{F} \cup\{\mathcal{M}\}$. Hence $I \in \mathcal{F}$ if $I \neq \mathcal{M}$. 
Now assume that $I=\cup_{\zeta<\sigma} I_{\zeta}$. If $\Omega_{n} \cap[0, \sigma[$ is cofinal in $[0, \sigma[$ for some $n \geq 1$, then $I \in \mathcal{V}\left(\mathcal{F}_{n}\right) \subset \mathcal{F} \cup\{\mathcal{M}\}$.

If $I=\cup_{\zeta<\sigma} I_{\zeta}$, and if $\Omega_{n} \cap[0, \zeta[$ is not cofinal in $[0, \zeta[$ for any $n \geq 1$, then $\sigma$ is a limit ordinal, and for every $m \geq 1$ there exist $\delta<\sigma$ such that $n(\zeta) \geq m$ for $\delta<\zeta<\sigma$.

Let $a \in I_{\sigma}$. The set $\left\{\theta_{I_{\zeta}}(a)\right\}_{\zeta<\sigma}$ is finite, so we have

$$
\lim _{m \rightarrow \infty} \max _{\zeta<\sigma}\left\|\theta_{I_{\zeta}}(a)\right\|_{m}=0,
$$

and there exists $m_{1} \geq 1$ such that $\left\|\theta_{I_{\zeta}}(a)\right\|_{m}<\epsilon / 3$ for every $\zeta<\sigma$ and every $m \geq m_{1}$. Let $\delta<\sigma$ such that $n(\eta) \geq m_{1}$ for $\delta<\eta<\sigma$, and let $u \in I \backslash I_{\delta}$. Applying 2.3 to the family $\left\{I_{\zeta}\right\}_{\zeta \leq \delta} \cup \mathcal{M}_{\omega_{1}}$, we obtain

$$
\lim \sup _{n \rightarrow+\infty} \max _{\zeta \leq \delta} q_{\zeta}\left(a-a u_{n}\right)=0 .
$$

Hence there exists $m_{2} \geq m_{1}$ such that $\max _{\zeta \leq \delta} q_{\zeta}\left(a-a u_{n}\right)<\epsilon$ for every $n \geq m_{2}$, and we can assume that $\left\|u_{n}\right\|_{1}<2$ for $n \geq m_{2}$, so that we have, for $\delta<\zeta<\sigma, n \geq m_{2}$

$$
q_{\zeta}\left(a-a u_{n}\right) \leq q_{\zeta}(a)\left(1+q_{\zeta}\left(u_{n}\right)\right) \leq q_{\zeta}(a)\left(1+\left\|u_{n}\right\|_{1}\right)<\epsilon .
$$

We thus see that $q_{\mathcal{F}}\left(a-a u_{n}\right)<\epsilon$ for $n \geq m_{2}$. Hence $I=\cup_{\zeta<\sigma} I_{\zeta}=I_{\sigma} \in$ $\mathcal{F} \cup\{\mathcal{M}\}$, and $I \in \mathcal{F}$ if $I \neq \mathcal{M}$.

In the case where $A=\mathbb{C}_{\omega_{1}}$, we obtain the following result, which does not depend on the continuum hypothesis.

Theorem 2.5 Let $\mathcal{F}$ be a chain of prime ideals of $\mathbb{C}_{\omega_{1}}$ such that $\mathcal{F} \cup\left\{\mathcal{M}_{\omega_{1}}\right\}$ is almost stable under unions. Then there exists an algebra seminorm on $\mathbb{C}_{\omega_{1}}$ such that the set of nonmaximal prime ideals of $\mathbb{C}_{\omega_{1}}$ which are closed with respect to q equals $\mathcal{F}$.

We do not know whether the set of prime ideals of $\mathbb{C}_{\omega_{1}}$ which are closed with respect to an algebra seminorm on $\mathbb{C}_{\omega_{1}}$ is necessarily almost stable under unions. The following simple observation shows that this is indeed the case for algebra seminorms constructed via the method used in the proof of lemma 2.4.

Proposition 2.6 Let $(E,\|\|$.$) be a normed vector space, and let \mathcal{F}$ be a chain of linear subspaces of $E$ stable under unions. Assume that there exists a family $\left(P_{F}\right)_{F \in \mathcal{F}}$ of endomorphisms of $E$ satisfying $\operatorname{Ker}\left(P_{F}\right)=F$ such that $P_{F_{1}} \circ P_{F_{2}}=$ $P_{F_{2}}$ for $F_{1}, F_{2} \in \mathcal{F}, F_{1} \subseteq F_{2}$. Let $\left(\|\cdot\|_{n}\right)_{n \geq 1}$ be a sequence of norms on $E$, and let $\mathcal{H}$ be the set of linear spaces $F \in \mathcal{F}$ such that $P_{F}:(E,\|\|.) \rightarrow\left(E,\|.\|_{n}\right)$ is bounded for some $n \geq 1$. Then $\mathcal{H}$ is almost stable under unions, and $F$ is a closed subspace of $(E,\|\|$.$) for every F \in \mathcal{H}$.

Proof: Clearly, $F=P_{F}^{-1}(0)$ is closed in $(E,\|\|$.$) for every F \in \mathcal{H}$. For $m, n \geq$ 1 set $\mathcal{H}_{m, n}:=\left\{F \in \mathcal{H} \mid\left\|P_{F}(x)\right\|_{n} \leq m\|x\| \forall x \in E\right\}$, so that $\mathcal{H}=\cup_{m, n \geq 1} \mathcal{H}_{m, n}$. Let $\left(F_{\lambda}\right)_{\lambda \in \Lambda}$ be a family of elements of $\mathcal{H}_{m, n}$, and set $F:=\cup_{\lambda \in \Lambda} F_{\lambda}$, so that 
$F \in \mathcal{F}$. Let $x \in E$. Then $x-P_{F}(x) \in \operatorname{Ker}\left(P_{F}\right)=F$, and so $x-P_{F}(x) \in F_{\lambda}$ for some $\lambda \in \Lambda$. We have

$$
P_{F_{\lambda}}(x)-P_{F}(x)=P_{F_{\lambda}}-\left(P_{F_{\lambda}} \circ P_{F}\right)(x)=P_{F_{\lambda}}\left(x-P_{F}(x)\right)=0 .
$$

Hence $\left\|P_{F}(x)\right\|_{n}=\left\|P_{F_{\lambda}}(x)\right\|_{n} \leq m\|x\|$ and $F \in \mathcal{H}_{m, n}$, which shows that $\mathcal{H}_{m, n}$ is stable under unions.

The maps $\left(\theta_{I}\right)_{I \in \mathcal{P}_{\omega_{1}}}$ satisfy the condition $\theta_{I_{1}} \circ \theta_{I_{2}}=\theta_{I_{2}}$ if $I_{1}$ and $I_{2}$ are prime ideals of $\mathbb{C}_{\omega_{1}}$ such that $I_{1} \subseteq I_{2}$. Let $\left(\|\cdot\|_{n}\right)_{n \geq 1}$ be any nonincreasing family of algebra norms on $\mathbb{C}_{\omega_{1}}$, let $\mathcal{F}$ be any well-ordered family of nonmaximal prime ideals of $\mathbb{C}_{\omega_{1}}$, and let $\delta: \mathcal{F} \rightarrow \mathbb{N}$ be an application. Let $\mathcal{I}_{q}$ be the set of prime ideals closed with respect to the algebra seminorm defined for $u \in \mathbb{C}_{\omega_{1}}$ by the formula

$$
q(u)=\max _{I \in \mathcal{F}}\left\|q_{I}(u)\right\|_{\delta(I)} .
$$

If follows from the proposition that $\mathcal{I}_{q}$ contains a family $\tilde{\mathcal{F}} \supset \mathcal{F} \cup\left\{\mathcal{M}_{\omega_{1}}\right\}$ which almost stable under unions. Hence other ideas would be needed to construct, if possible, an algebra seminorm $q$ on $\mathbb{C}_{\omega_{1}}$ such that $\mathcal{I}_{q} \cup\left\{\mathcal{M}_{\omega_{1}}\right\}$ is not almost stable under unions.

Denote by $\operatorname{Prim}(q)$ the set of nonmaximal prime ideals of $\mathcal{C}(K)$ which are closed with respect to an algebra seminorm $q$ on $\mathcal{C}(K)$. We also deduce from lemma 2.4 the following result.

Theorem 2.7 (CH) Let $K$ be an infinite compact space, and let $\mathcal{F}$ be a wellordered chain of nonmaximal prime ideals of $\mathcal{C}(K)$ such that $\left|\mathcal{C}(K) / I_{0}\right|=2^{\aleph_{0}}$, where $I_{0}$ denotes the smallest element of $\mathcal{F}$, and let $\mathcal{M}$ be the maximal ideal of $\mathcal{C}(K)$ containing the elements of $\mathcal{F}$.

If $\mathcal{F} \cup\{\mathcal{M}\}$ is almost stable under unions, then there exists a discontinuous algebra norm $q$ on $\mathcal{C}(K)$ such that $\mathcal{F}=\operatorname{Prim}(q)$.

Proof: There exists a one-to-one homomorphism $\phi: \mathcal{C}(K) / I_{0} \rightarrow \mathbb{C}_{\omega_{1}}$. Set $A=\phi\left(\mathcal{C}(K) / I_{0}\right)$. Denote by $\mathcal{C}_{\mathbb{R}}(K)$ the algebra of continuous real valued functions on $K$, and set $J=I_{0} \cap \mathcal{C}_{\mathbb{R}}(K)$. Let $\pi: \mathcal{C}(K) \rightarrow \mathcal{C}(K) / I_{0}$ be the canonical surjection. It is a standard fact that the quotient order on $\mathcal{C}_{\mathbb{R}}(K) / J$ is a linear order. Let $f, g \in \mathcal{C}(K)$. If, say, $\pi(|f|) \leq \pi(|g|)$, then $\pi(f)^{2} \in \pi(g) \mathcal{C}(K) / I$, see for example lemma 2.1 of [22]. It is also a standard fact that the equation $x^{n}=a$ has a solution in $\mathcal{C}(K) / I_{0}$ for every $a \in \mathcal{C}(K) / I_{0}$ (more generally if $I$ is a prime ideal of $\mathcal{C}(K)$ the equation $x^{n}+a_{n-1} x^{n-1}+\ldots+a_{0}=0$ has a solution in $\mathcal{C}(K) / I$ for $a_{0}, \ldots, a_{n-1} \in \mathcal{C}(K) / I$, use for example exercise 13A of [15] to adapt to the complex case the argument used in the proof of theorem 13.4 of [15] to prove an analogous result in the real case for $n$ odd). So $A$ satisfies conditions (i) and (ii) of lemma 2.3.

If $p$ is any algebra seminorm on $A$, then $q:=p \circ \phi \circ \pi$ is an algebra seminorm on $\mathcal{C}(K)$, and it follows from [24] or [8] that $f$ belongs to the closure of $f^{2} \mathcal{C}(K)$ with respect to $q$ for every $f \in \mathcal{C}(K)$. Hence $a$ belongs to the closure of $a^{2} A$ 
with respect to $p$ for every $a \in A$, and every ideal of $A$ which is closed with respect to $p$ is semiprime, hence prime. So we can apply lemma 2.4 to $A$ and to the family $\mathcal{G}:=\{(\phi \circ \pi)(I)\}_{I \in \mathcal{F}}$. We obtain a seminorm $p$ on $A$ such that the set of nonmaximal ideals of $A$ which are closed with respect to $p$ equals $\mathcal{G}$ (in fact $p$ is a norm since $\{0\}=(\phi \circ \pi)\left(I_{0}\right)$ is closed with respect to $\left.p\right)$. Clearly, the set of nonmaximal ideals of $\mathcal{C}(K)$ which are closed with respect to $q:=p \circ \phi \circ \pi$ equals $\mathcal{F}$. Now set $\tilde{q}(f)=\max \left(q(f),\|f\|_{K}\right)$ for $f \in \mathcal{C}(K)$. Then $\tilde{q}$ is an algebra norm on $K$, and it is well known that $\operatorname{Prim}(\tilde{q})=\operatorname{Prim}(q)$ (see for example [8]), so that $\operatorname{Prim}(\tilde{q})=\mathcal{F}$.

If $\mathcal{U}$ is a family of nonmaximal prime ideals of $\mathcal{C}(K)$, denote by $\mathcal{M}_{\mathcal{U}}$ the set of maximal ideals of $\mathcal{C}(K)$ which contain some element of $\mathcal{U}$. Theorem 2.7 suggests the following conjecture

Conjecture $1(\mathrm{CH})$ Let $\mathcal{U}$ be a family of nonmaximal prime ideals of $\mathcal{C}(K)$ satisfying the three following properties

(i) every sequence of elements of $\mathcal{U}$ has a pseudo-finite subsequence,

(ii) $\mathcal{U} \cup \mathcal{M}_{\mathcal{U}}$ is almost stable under unions,

(iii) $|\mathcal{C}(K) / I|=2^{\aleph_{0}}$ for every minimal element $I$ of $\mathcal{U}$.

Then there exists an algebra norm on $\mathcal{C}(K)$ such that $\mathcal{U}=\operatorname{Prim}(q)$.

It is known that the "continuity ideal" of a discontinuous homomorphism from $\mathcal{C}(K)$ can be written as the intersection of a "relatively compact" family of nonmaximal prime ideals in the sense of [21], or equivalently, is a pure semiprime Badé Curtis ideal in the sense of definition 1.2 of [12] (details about this equivalence can be found in [13]). If the conjecture were true, it would imply that, conversely, every ideal $I$ of $\mathcal{C}(K)$ satisfying $|\mathcal{C}(K) / I|=2^{\aleph_{0}}$ which can be written as the intersection of a "relatively compact" family of nonmaximal prime ideals is the continuity ideal of some discontinuous homomorphism from $\mathcal{C}(K)$, which would allow to remove the countability condition of therorem 6.7 (ii) of [21] and show that the continuity ideals of discontinuous homomorphisms from $\mathcal{C}(K)$ are exactly the pure semiprime Badé-Curtis ideals. This result would have some heuristic interest since the fact that continuity ideals are pure semiprime BadéCurtis ideals is a consequence of the two main tools of automatic continuity theory, the main boundedness theorem of Badé-Curtis and Sinclair's stability lemma. These tools would thus provide all possible information about discontinuous homomorphisms from $\mathcal{C}(K)$ if the continuum hypothesis is assumed.

Now let $I$ be a prime ideal of $\mathcal{C}(K)$, and let $\mathcal{J}(I)$ be the intersection of all minimal prime ideals of $\mathcal{C}(K)$ contained in $I$. Following definition 5.8 of [12], we will say that a subalgebra $B$ of $\mathcal{C}(K)$ is a lifting of the quotient algebra $\mathcal{C}(K) / I$ if $B$ satisfies the two following conditions

(1) $B \cap I=\mathcal{J}(I)$

(2) $\mathcal{C}(K)=B+I$.

If $I$ is a minimal prime ideal of $\mathcal{C}(K)$ then $\mathcal{C}(K)$ itself is obviously a lifting of $\mathcal{C}(K) / I$, and if $\mathcal{M}_{\tau}=\{f \in \mathcal{C}(K) \mid f(\tau)=0\}$ is a maximal ideal of $\mathcal{C}(K)$ then 
the algebra of functions constant on some neighbourhood of $\tau$ is a lifting of the one-dimensional quotient algebra $\mathcal{C}(K) / \mathcal{M}_{\tau}$.

It is shown in [13] that the quotient algebra $A_{I}$ does possess a lifting for every prime ideal $I$ of $\mathcal{C}(K)$. More precisely let $I$ be a prime ideal of $\mathcal{C}(K)$, and let $J$ be a prime ideal of $\mathcal{C}(K)$ containing $I$. It proved in [13] that every lifting of $\mathcal{C}(K) / I$ contains a lifting of $\mathcal{C}(K) / J$, and that, conversely, every lifting of $\mathcal{C}(K) / J$ is contained in some lifting of $\mathcal{C}(K) / I$. This suggests the following problem.

Problem 1 Let $I$ be a prime ideal of $\mathcal{C}(K)$ and let $\mathcal{F}$ be the set of prime ideals of $\mathcal{C}(K)$ containing $I$. Does there exist a family $\left\{B_{J}\right\}_{J \in \mathcal{F}}$ of subalgebras of $\mathcal{C}(K)$ which possesses the following properties?

(i) $B_{J}$ is a lifting of the quotient algebra $\mathcal{C}(K) / J$ for every $J \in \mathcal{F}$.

(ii) If $J_{1} \in \mathcal{F}, J_{2} \in \mathcal{F}$, and if $J_{1} \subseteq J_{2}$, then $B_{J_{2}} \subseteq B_{J_{1}}$.

A positive answer to this problem, at least in the case where $|\mathcal{C}(K) / I|=2^{\aleph_{0}}$, would imply a result analogous to lemma 2.2 for the quotient algebra $\mathcal{C}(K) / I$, which would give a direct proof of theorem 2.7. This would also open the gate to a proof of conjecture 1 . As mentioned above, this would give a complete characterization under $\mathrm{CH}$ of ideals $I$ of $\mathcal{C}(K)$ satisfying $|\mathcal{C}(K) / I|=2^{\aleph_{0}}$ which are equal to the continuity ideal of some discontinuous homomorphism from $\mathcal{C}(K)$. Unfortunately, the author was not able so far to solve this question, despite periodic attempts to solve a slightly weaker form of problem 1 during the last 35 years. The answer might depend on axioms of set theory.

Notice that since a maximal ideal of $l^{\infty}$ contains a unique minimal prime ideal, and since the quotient algebra $l^{\infty} / I$ is isomorphic to $\mathbb{C}_{\omega_{1}}$ for every nonmaximal minimal ideal of $l^{\infty}$, it follows from lemma 2.2 that the answer to problem 1 is positive for $\beta \mathbb{N}$ if $\mathrm{CH}$ is assumed. We do not know the answer to this problem for $\beta \mathbb{N}$ in $\mathrm{ZFC}$.

\section{References}

[1] G.R. ALLAN, 'Embedding the algebra of all formal power series in a Banach algebra', Proc. London Math. Soc. 25 (1972), 329-340.

[2] W.G. BADE and P.C. CURTIS, 'Homomorphisms of commutative Banach algebras', Amer. J. Math. 82 (1960), 589-608.

[3] H.G. DALES, 'A discontinuous homomorphism from $\mathcal{C}(X)$ ', Amer. J. Math. 101 (1979), 647-734.

[4] H.G. DALES, Banach algebras and automatic continuity, (London Mathematical Society Monographs, vol 24, The Clarendon Press, Oxford, 2000).

[5] H.G. DALES and J. ESTERLE, 'Discontinuous homorphisms from $\mathcal{C}(X)$ ', Bull. Amer. Math. Soc. 83 (1977), 257-259. 
[6] H.G. DALES and W. H. WOODIN, An introduction to independence for analysts, (London Mathematical Society Lecture Notes Series, vol. 115, Cambridge University Press, Cambridge, 1987).

[7] H.G. DALES and W. H. WOODIN, Super-real fields: totally ordered fields with additional structure, (London Mathematical Society Monographs, Vol 14, Clarendon Press, Oxford, 1996).

[8] J. ESTERLE, 'Semi-normes sur $\mathcal{C}(K)$ ', Proc. London Math. Soc. 36 (1978), $27-45$.

[9] J. ESTERLE, 'Sur l'existence d'un homomorphisme discontinu de $\mathcal{C}(K)$ ', Proc. London Math. Soc. (3) 36 (1978), 46-58.

[10] J. ESTERLE, 'Injection de semigroupes divisibles dans des algèbres de convolution et construction d'homomorphismes discontinus de $\mathcal{C}(K)^{\prime}$ ', Proc. London Math. Soc. 36 (1978), 59-85.

[11] J. ESTERLE, 'Universal properties of some commutative radical Banach algebras', J. Reine Angew. Math. 321 (1981), 1-24.

[12] J. ESTERLE, 'Kaplansky's and Michael's problems: a survey', Ann. Func. An. 3 (2012), 66-88.

[13] J. ESTERLE, 'Closed prime ideals for discontinuous algebra seminorms on $\mathcal{C}(K)^{\prime}$, preprint.

[14] R. FRANCKIEWICZ and P. ZBIERSKI, Hausdorff gaps and limits, (Van Nostrand, New-York, 1994).

[15] L. GILLMAN and M. JERISON, Rings of continuous functions, (The University Series in Higher Mathematics, D. Van Nostrand Co., Inc., Princeton, New Jersey- Toronto-London-New-York, 1960).

[16] H. HAHN, Uber die nichtarchimedischen Großensysteme, Sitzungsberichte der Kaiserlichen Akademie der Wissenschaften, Wien, Mathematisch Naturwissenschaftliche Klasse (Wien. Ber.) 116 (1907), 601-655.

[17] I. KAPLANSKY, 'Normed algebras', Duke Math. J. 16 (1949), 399-418.

[18] S. MAC LANE, 'The universality of power series fields', Bull. Amer. Math. Soc. 45 (1939), 888-890.

[19] H. L. PHAM, 'The kernels of radical homomorphisms and intersections of prime ideals', Trans. A.M.S. 360 (2008), 1057-1088.

$[20]$ H. L. PHAM, 'Uncountable families of prime $z$-ideals in $\mathcal{C}_{0}(\mathbb{R})$ ', Bull. London Math. Soc. (2) 41 (2009), 354-366.

[21] H. L. PHAM, 'The kernels and continuity ideals of homomorphisms from $\mathcal{C}_{0}(\Omega)^{\prime}$, J. Aust. Math. Soc. 88 (2010), 103-130. 
[22] D. RUDD, 'On two sum theorems for ideals of $C(X)$ ', Michigan Math. J. 17 (1970), 139-141.

[23] W. SIERPINSKY, 'Sur une propriété des ensembles ordonnés', Fund. Math. 36, 1949, 56-67.

[24] A.M. SINCLAIR, 'Homomorphisms from $\mathcal{C}_{0}(\mathbb{R})$ ', J. London Math. Soc. 11 (1975), 165-174.

[25] A.M. SINCLAIR, Automatic continuity of linear operators, ( London Mathematical Society Lecture Note Series, No. 21, Cambridge University Press, Cambridge, 1976).

[26] W. H. WOODIN, 'A Discontinuous Homomorphism from $C(X)$ without CH', J. London Math. Soc. 48 (1993), 299-315.

Jean Esterle

IMB, UMR 5251

Université de Bordeaux

351, cours de laLibération

33405 - Talence (France)

esterle@math.u-bordeaux1.fr 